\title{
A Quantum Biomechanical Basis for Near-Death Life Reviews
}

\author{
Thomas E. Beck, Ph.D. \\ Janet E. Colli, Ph.D. \\ Seattle, WA
}

\begin{abstract}
Near-death life reviews pose a challenge to current memory research in terms of the sheer amount of instantaneous and empathetic information recall. Advances in quantum physics, biomechanics, holographic information theory, and consciousness studies support for the first time a fully realizable quantum biomechanical basis for near-death life reviews. We introduce the unifying paradigm of the quantum hologram as a non-local carrier of information. We further investigate the interrelated phenomena of non-local communications, and the electromagnetic zero-point field. Recent confirmation of the zero-point field lends credibility to vast memory storage capabilities outside the physical body. Microtubules are considered to be key components in non-local, quantum processes critical to human consciousness. Discovery of the liquid crystalline nature of the human body provides further support for our model. Microtubules, deoxyribonucleic acid (DNA), and the entire brain are described as communicating non-locally with virtually unlimited memory storage capacity.
\end{abstract}

KEY WORDS: near-death experience; life review; memory; non-local communication; quantum hologram; microtubules.

It has been said that the foundation of modern psychiatry was formed by the study of the impact of traumatic experience (van der Kolk and van der Hart, 1991). One issue of focus for memory researchers is traumatic memory retrieval. Do overwhelming experiences leave fixed and indelible memories? While overwhelming experiences make up only a portion of what is recalled during near-death life reviews, it is instructive to

Thomas E. Beck, Ph.D., is a transpersonal psychotherapist, and is on the advisory board of Intellergy Institute, founded to derive the transformations between information and physical reality. Janet E. Colli, Ph.D., is a transpersonal psychotherapist and consciousness researcher. Reprint requests should be addressed to Drs. Beck and Colli at the Good Shepherd Center, 4649 Sunnyside Avenue North, Suite 341, Seattle, WA 98103; e-mail: becolli@mindspring.com. 
consider the perspective of current memory research. For if even traumatic memories are not fixed and indelible, how can other elements of the near-death life review be said to be accurate, much less all elements of the life review? We begin with a focused review of memory research, and then explicate three aspects of near-death life reviews that would be problematic to memory researchers of ordinary and even overwhelming experiences.

\section{Introduction to Memory Research}

Non-traumatic memories have been shown to be subject to alteration. The "misinformation effect," that is, how exposure to misinformation induces memory distortion, has been extensively studied in the laboratory. Elizabeth Loftus and Hunter Hoffman (1989) argued that misinformation can impair the original memory traces themselves. Memories have been found to be more easily modified when the passage of time allows for the original memory to fade:

Give us a dozen healthy memories, well-formed, and our own specified world to handle them in. And we'll guarantee to take any one at random and train it to become any type of memory that we might select-hammer, screwdriver, wrench, stop sign, yield sign, Indian chief-regardless of its origin or the brain that holds it. (Loftus and Hoffman, 1989, p. 103)

Loftus has most recently dedicated herself to the creation of "false" memories, experimentally "implanting" memories for nonexistent events that would supposedly have been traumatic had they occurred, such as being lost in a shopping mall as a small child (1997).

However, there are significant differences between such memories and the perceptions characteristic of posttraumatic stress disorder (PTSD). The opposing side of the debate focuses on the initial form traumatic memories take, that is, dissociated mental imprints of sensory and affective elements in the visual, auditory, olfactory and kinesthetic channels. Trauma is said to be "speechless terror," with narrative memory emerging only over time (van der Kolk and Fisler, 1995). One prominent researcher of traumatic stress has gone so far as to write:

As of early 1995, I could find no published accounts in the scientific literature of intrusive traumatic recollections of traumatic events in patients suffering from PTSD that had become distorted over time, naturalistically, or by manipulation, either in an experimental or in a clinical setting. (van der Kolk, McFarlane, and Weisaeth, 1996, p. 282) 
However, inconsistent recall for specific features of combat memory has recently been demonstrated, thus challenging the notion that there is an indelible fixation in the mind of traumatic events (Southwick, Morgan, Nicolaou, and Charney, 1997).

Memory researchers have thus sought a closer look at brain regions that are activated in the retrieval of memories. The application of brain imaging techniques such as magnetic resonance imaging (MRI) allows the examination of the neuroanatomical correlates of both veridical and illusory memories (Gauthier, Hayward, Tarr, Anderson, Skudlarski, and Gore, 2002). Positron emission tomography (PET) scans measure changes in blood flow in the brain that indicate neural activity. PET scans of subjects who merely listened to lists of words showed that "accurate memories appear to retrieve sensory details that are not available to us in illusory memories" (Begley, 1996, p. 64; Schacter, Reiman, Curran, Yun, Bandy, McDermott, and Roediger, 1996). The "true" memories activated areas in the superior temporal lobe, the region that processes sounds of recently heard words. The implication is that retrieval of veridical memories activates the brain regions that originally processed the perceptual stimuli during encoding (the auditory cortex in this case).

However, sensory signatures interpreted as an expression of a distinctive memory trace present during the original event may not be readily traceable in the slower response measures obtained with MRI and PET scans. Such transient brain activity has been isolated with event-related brain potentials (ERPs) recorded with scalp electrodes. One study demonstrated differences in event-related brain potentials at the time of retrieval for true versus false memories (Fabiani, Stadler, and Wessels, 2000). Brain activity associated with memory for traumatic and emotionally charged events is only just beginning to be studied. But as memory is influenced by the biochemistry of the stress response, markers for memory veracity are being found in measurable physiological changes in victims of abuse (Cotton, 1994).

Yet the search for physiological markers of memory veracity is at odds with what many near-death researchers take for granted: that is, the veracity of entire life reviews. After all, the notion of the brain as a virtual "videorecorder" has been seriously challenged. Wilder Penfield's well-known work on electrical stimulation of certain brain sites during surgery on epileptic patients during the 1940s has since been reevaluated. Note Penfield's 1969 account of the stable nature of the flashbacks: 
It is clear that the neuronal action that accompanies each succeeding state of consciousness leaves its permanent imprint on the brain. The imprint, or record, is a trail of facilitation of neuronal connections that can be followed again by an electric current many years later with no loss of detail, as though a tape recorder had been receiving it all. (1969, p. 165)

However, a closer look at this widely cited research shows that these responses were relatively rare ( 3.0 to 7.7 percent of cases), and were vague descriptions, more likely reconstructions or inferences rather than actual memories (Loftus and Loftus, 1980). Indeed, by 1975 Penfield could be seen as more conservative, and did not regard the electrically stimulated impressions perceived by subjects as real memories, but rather as artificial.

\section{Near-Death Life Reviews}

Given this onslaught against the veracity of memory itself, how can near-death researchers defend the veracity of near-death life reviews, an experience said to entail all significant memories during a lifetime? Even if overwhelming experiences are remembered as dissociated sensory elements and not subject to distortion, as Bessel van der Kolk, Alexander McFarlane, and Lars Weisaeth (1996) argued, typical neardeath life reviews are remembered as narrative, explicit memories. PET scans and MRIs of near-death experiencers (NDErs) during later recall of their life review could help resolve the issue of veridicality. However, the search for a physical substrate for near-death life reviews is also critical.

Melvin Morse and Paul Perry (1992) linked life reviews to the Sylvian fissure of the right temporal lobe, though they have been faulted (Wade, 1996) for relying on Penfield's work. They hypothesized an excitation of the body's electromagnetic field at death, which revives the fading right temporal lobe to record the NDE. However, few memory theorists would hold the right temporal lobe, normally associated with long-term memories, capable of recording the NDE accurately if it entails an entire life review. Also, some NDErs have no measurable brain activity (Rosenthal and Larson, 1978; Sabom, 1998). "Saying that the Sylvian fissure is capable of producing certain percepts under special conditions during life is very different from assuming that part of the brain dominates consciousness after death and that it functions in the same manner" (Wade, 1996, p. 232). Brain imaging techniques during later 
recall of the near-death life review may provide a means for testing the Sylvian fissure hypothesis, as the formation of memories is a function of the brain regions activated, and recall would conceivably activate those same regions.

Even so, Morse's extension of the Sylvian fissure argument is at the forefront of the attempt to account for paranormal experiences and abilities arising from the NDE. But though Morse went well beyond Penfield's conclusion of strict localization of brain function, we believe he did not fully account for three singular aspects of near-death life reviews. We will present a non-localized memory theory evolved from Karl Pribram's view that the memory engram may be an interference pattern similar to that made by a laser in a hologram.

Prior to Morse, researchers focused on an underlying neurological mechanism for near-death life reviews, though Russell Noyes and Roy Kletti (1977) also cited Penfield's work as evoking vivid memory recall, which has not been borne out. Gordon Greene, in his search for a viable causal factor, added the additional concept of hyperspace, "any space with more than three dimensions" (Greene, 1981, p. 121). As time spatializes into a fourth dimension, we are said to be able to perceive hyperspatially and spontaneously, the whole of our lives. Though the concept of hyperspace correlates with modern-day physicists' concept of nth-dimensional space, Greene himself countenanced "the difficulty of obtaining more than just circumstantial evidence that higher spaces actually exist" (1981, p. 129).

More recently, Linz Audain (1999) attempted to link neurobiological concepts to the possibility of the existence of a hyperspace. Yet his attempt to spark "a long debate within the scientific community as to the nature of the interaction that exists between the neurological and the metaphysical" (Audain, 1999, p. 104) is likely to fail. Few physical scientists consider metaphysical subjects worthy of serious dialogue. As recently as 1994, an article in a respected physics journal (Stapp, 1994) cited an article in the Journal of Parapsychology in presenting a theoretical model that would allow for causal anomalies incompatible with well-established principles of physics. However, citing data from a parapsychology journal provoked opposition from the physics journal's editor, who expressed his strong opinion that henceforth such papers should not be allowed (H. Stapp, personal communication, February 8, 2002). Carl Becker (1995) has duly faulted Kenneth Arnette (1999) for applying scientific concepts to the nonmaterial substance he called "essence." Furthermore, the necessity of a dualistic approach has long been questioned (Krishnan, 1996). We intend to apply scientific 
concepts in a non-dualistic manner to explain the existence of consciousness phenomena on a macroscopic level.

Given the malleable nature of non-traumatic memories, perhaps NDE researchers can profit from the lesson learned by memory researchers: that is, to differentiate between normal memory and memory in extreme altered states. For possibly near-death life reviews are not memories in the usual sense of the word, as suggested by Raymond Moody: "This review can only be described in terms of memory, since that is the closest familiar phenomenon to it, but it has characteristics which set it apart from any normal type of remembering" (1975, p. 64).

Three singular aspects of near-death life reviews cast doubt on their credibility when considered solely in light of current memory research and classical physics: (a) the sheer amount of sensory information and information recall; (b) the apparently instantaneous, panoramic presentation of memories; (c) the review of the experience as perceived by others. Advances in science support for the first time a theoretical basis for the underlying physical mechanism that would account for these three singular aspects of near-death life reviews. Examples of each will serve to illustrate the vast amount of data apparently in memory storage, and how the transparency of ego boundaries seems to allow access to collective memory.

\section{Amount of Information Recalled}

NDErs describe exactly what memory theorists such as Loftus have attempted to discredit: that is, that human memory can function like a videorecorder. NDErs frequently use terms like a "movie camera" or "tape recorder" to express that every single thing they experienced in their lives was recorded, though it may not have registered in consciousness at the time:

It was like watching my life from start to finish on an editing machine stuck in fast forward. The review took me from my conception, which felt like the blackness I experienced after my out-of-body experience, through my childhood, to adolescence, into my teens, and through my near-death experience over again. I saw my life. I relived my life. I felt everything I ever felt before. When I say "everything," I mean every cut, pain, emotion and sense associated with that particular time in my life. (Ring and Valarino, 1998, p. 22)

I not only relived [this incident] in my life review, but I relived every exact thought and attitude - even the air temperature and things that I couldn't have possibly measured when I was eight years old. For example, I wasn't aware of how many mosquitoes were in the area. In 
the life review, I could have counted the mosquitoes. Everything was more accurate than could possibly be perceived in the reality of the original event. (Ring and Valarino, 1998, p. 170)

\section{Instantaneous, Panoramic Presentation of Memories}

Whether or not a temporal order of events is remembered, the threedimensional, panoramic review occurs in what seems like an instant of time:

With regard to the question of time, everything happened instantaneously.... It is like an explosion, it is all there. When my life went before my eyes, it was not from my earliest memory at thirteen months. There was an enormous TV screen in front of me... Way over on the left was my memory at thirteen months, and way over on the right was July, 1972, age thirty-eight. Everything in between was right there and I could see the whole thing, all at the same instant. (Ring and Valarino, 1998, p. 150)

\section{Review of the Experience as Perceived by Others}

The review is experienced from the perspective of all lives that intersected with one's own, from purely personal interactions to the transpersonal or collective level of consciousness:

I rethought every thought, I reexperienced every feeling, as it happened, in an instant. And I also felt how my actions, or even just my thoughts, had affected others. When I had passed judgment on someone else, I would experience myself doing that.... Multitudinous actions or thoughts, derived from my own meanness, unkindness, or anger, caused me to feel the consequent pains of the other people. I experienced this even if at the time I had hurt someone, I had chosen to ignore how that would affect them. And I felt their pain for the full length of time they were affected by what I had done. (Ring and Valarino, 1998, pp. 158-159)

I learned that time as we think of it doesn't exist, nor does the separation between us.... in fact, it was almost as if there was no "other." I say almost because I had self-awareness, but knew my awareness lived within an intricate pattern that existed eternally, everywhere. (Ring and Valarino, 1998, p. 176)

I experienced all of my experiences not only from my perspective but also from everyone else's, even those I didn't even know or interact with.... I then crossed the threshold of the birth of this body and this process of Review accelerated and I relived all former lives of my particular "soul," if you will... Then I lived all lives being lived on this 
planet. And finally, all lives being lived throughout the Universe, all the way to the Ultimate Awareness of All Creation. It was a truly incredible, indescribable experience. (Colli, 2001)

If veridical, these three aspects of near-death life reviews highlight the limited perspective of current memory research. The challenge such reviews pose cannot be overemphasized. However, perhaps these reviews are problematic only when considered in light of classical physics, and the laws underlying the ordinary world of macroscopic objects. The following sections examine how "extraordinary" experiences of ordinary humans take us beyond the scope of classical physics.

\section{Quantum Biomechanics}

In light of recent scientific developments, the experience of neardeath life reviews can be seen to acquire new legitimacy. With the emergence of quantum biomechanics, a new understanding of the mechanism of life reviews may be articulated. Two mutually related concepts are crucial to the application of quantum biomechanics to the functioning of the human body: quantum coherence and non-local communication. We begin by describing lasers in order to explain the relevance these concepts have for consciousness, for quantum coherence and nonlocal communication occur within structures in the physical human body.

\section{Quantum Coherence}

This natural physical phenomenon involves large numbers of light (or matter) particles that cooperate collectively in a single state. The intense, narrow beam of a laser exemplifies quantum coherence. All of the emitted light particles, or photons, oscillate together at the same frequency and phase, resulting in a light beam of a single color. Driven by external stimulation, the laser reaches a critical energetic threshold, above which it undergoes a non-linear phase transition, an abrupt jump to a higher energy level. In laser light, all the photons lack individual identities and thus are said to be in the same quantum state (Marshall, 1989). By contrast, incandescent and fluorescent lamps emit incoherent light in all directions, in a broad spectrum of frequencies, resulting in white light.

Lasers undergo a phase transition in which many photons become coherent and are thus described by the same mathematical wave function. In quantum mechanical terms, such systems of coherent excitations are 
called Bose-Einstein condensates. Bose-Einstein condensates demonstrate collective, macroscopic quantum states-macroscopic since the effects of some Bose-Einstein condensates can be directly observed, without the aid of magnification.

\section{Non-local Communication}

Quantum coherence between particles involves non-local communication, that is, interaction which is: (1) instantaneous, (2) independent of distance, and (3) impervious to shielding. Non-locality refers to processes in which signals propagate across any distance instantaneously. By contrast, signals that propagate in a finite period of time are called local signals; for example, the visible electromagnetic light to which our eyes respond (Goswami, 1993). Electromagnetic waves in the form of incoherent, white light, as in sunshine, or radio and television transmissions, exhibit none of these three characteristics. They traverse the distance between the transmitter and the receiver in a finite, measurable period of time; they diminish in intensity the farther they travel; and they can be blocked by appropriate shielding.

Non-locality is not to be confused with faster-than-light, or superluminal signal propagation. Superluminal signals (those propagating faster than 186,000 miles per second) would, despite their great velocity, still take many human lifetimes to cross our galaxy (Nimtz, 1998). Physicists have experimentally demonstrated non-local interaction between photons (Aspect, Dalibard, and Roger, 1982). These experiments demonstrate that non-local communication is possible across any arbitrarily large distance.

On the scale of human perception, evidence suggests that humans perceive by means of both local and non-local processes. For example, when viewing a nearby person, the eyes respond to electromagnetic waves, but the mind also seemingly responds to a non-local, instantaneous component (Mitchell, 1999). During remote viewing of the same person who is miles distant, only the non-local aspect is perceived by the viewer, which provides a dreamlike, unclear image. This is consistent with altered states of consciousness such as clairvoyance, precognition, and telepathy. The Central Intelligence Agency has engaged in "a multiyear, multi-site, multi-million-dollar effort to determine whether such phenomena as remote viewing 'might have any utility for intelligence collection' " (Puthoff, 1996, p. 64). Dean Radin (1997) summarized nonlocal experiments across a range of phenomena. His statistical analyses demonstrated the viability of non-local perception. Self-reports of 
near-death life reviews also suggest non-local perception, as they are said to be virtually instantaneous. All of the above experiences provide crucial support for the theory that the human body possesses the quantum biomechanical mechanisms necessary for non-local communication. However, until the current stage of scientific research, the scientific explanation for such phenomena has been lacking. Researchers are now beginning to connect these anecdotal reports to the underlying mechanisms on the molecular level.

On the molecular scale, non-local communication has been identified within the physical human body. We refer again to the laser-a Bose-Einstein condensate- whose core element can be a crystal. The resulting coherent beam of light carries enormous amounts of information, as in cable television. It has recently been discovered that living organisms, including much of the human body, are liquid crystalline in structure $(\mathrm{Ho}, 1999 ; 1998 \mathrm{~b})$. Liquid crystals are states of matter that encompass a wide range of fluidity, from solid crystal to semi-solid proteins to gel-like cellular fluids. Whereas the calcium phosphate crystals in bones are solid, the collagen in bone is semi-solid and is referred to as a liquid crystal. Biological organisms are largely composed of liquid crystalline materials such as connective tissue and cell membranes, plus other tissues that fill up the spaces between the organs. "The connective tissues of our body include the skin, bones, tendons, ligaments, cartilage, various membranes covering major organs and linings of internal spaces" (Ho, 1999). Liquid crystals, used extensively in calculator displays, possess properties that make them ideal for rapid intercellular communication. Within the coherent, liquid crystalline human body, intercommunication is instantaneous and non-local (Ho, 1998a).

As evidence of this, biomolecular quantum tunneling has been demonstrated in proteins and in deoxyribonucleic acid (DNA) in the human body (Stuchebrukhov, 1996; Wagenknecht, Rajski, Pascaly, Stemp, and Barton, 2001). Quantum tunneling refers to the instantaneous behavior of atomic-scale particles such as photons, electrons, protons, or hydrogen atoms that "jump" from point A to point B without traversing the distance in between. Physicist Guenter Nimtz (1999) has demonstrated quantum photon tunneling through a barrier, over a distance of about 5.5 inches. Actual signal traversal time within the barrier is instantaneous, regardless of barrier length (G. Nimtz, personal communication, April 6, 2002).

Another example of liquid crystalline substance is the intracellular cytoplasm, the fluid inside bodily cells. Microtubules are the principal organizing constituents of cytoplasm. Given that microtubules are 
found within most cells, we now address the role that microtubules play in non-local communication within the human body.

\section{Cellular Microtubules}

Bose-Einstein condensation can occur with both light particles, or photons, and matter particles, such as protons, neutrons, and electrons. While Bose-Einstein condensates comprised of matter have been demonstrated only at extremely low laboratory temperatures $\left(-459^{\circ} \mathrm{F}\right)$, lasers can operate at normal room temperatures $\left(72^{\circ} \mathrm{F}\right)$. Quantum coherence also occurs in living organisms, including the human body, which maintains an internal temperature of about $98.6^{\circ} \mathrm{F}$. Quantum coherence has been observed in specific biological structures called $\mathrm{mi}$ crotubule networks within nucleated cells (Hameroff, 1994). Evidence indicates that microtubules emit single photons of light and can be viewed as microscopic pulse-lasers within living cells (Popp, Li, Nagl, and Klima, 1983).

Microtubules are biological Bose-Einstein condensates that are considered to play an important role in human communications, memory recall, and learning. They may prove to be the fundamental elements of a non-local communication network that provide the basis for neardeath life reviews. A brief description of their properties will shed further light on these remarkable miniature communications networks.

As the name implies, microtubules are microscopic. They are selforganizing, tubular protein structures present in nearly all eukaryotic cells-that is, cells that have a nucleus. Eukaryotic cells include most cells in the human body and throughout nature. Microtubules form a complex skeleton-like structure that physically supports the entire cell, giving it shape and resilience. However, beyond their physical attributes, microtubules provide a complex communications network within each cell that is essential for the cell's overall functioning. Indeed, the microtubule network is sometimes referred to as the cell's "brain."

Part of the cell's microtubule structure is the microtubule organizing center, which runs the cell's "machinery" and organizes most, if not all, cell functions, including critical cell division. In some neural cells, the microtubules can be nearly one meter long and arranged in bundles of hundreds or thousands. They might be compared to fiber-optic cables used for telephone communications, which consist of numerous thin wires bundled together. For a sense of proportion, a microtubule 
one meter long would compare to a one-inch diameter garden hose over 600 miles long, though most microtubules are much shorter. On a molecular level, their complexity is incomprehensible. However, on a quantum level, the communication occurring within microtubules is a relatively simple process.

\section{Quantum Effects of Microtubules}

Communication at the level of microtubules has been described mathematically (Marcer and Schempp, 1997). A growing body of scientific knowledge supports the theory that microtubules possess three important properties related to intercellular and intracellular communications: (a) propagation of laser-like, coherent micropulses of light; (b) quantum, non-local information processing; and (c) emergent, collective, macroscopic properties arising from a critical level of coherence of quantum events.

Light Propagation. Microtubules propagate laser-like, coherent, single-photon micropulses of light that result from Bose-Einstein condensation. Evidence suggests that these individual micropulses of light effectively generate single-photon holograms, just as a laser beam, composed of countless individual photons, generates a hologram (Hirano and Hirai, 1986). If trillions of microtubules in the human body each create single-photon holograms, the amount of holographically encoded information may be effectively unlimited. However, unlike other BoseEinstein condensates, these occur at body temperature. Their energy source is the $98.6^{\circ}$ ambient heat bath of human cellular fluids, or cytoplasm, in which microtubules are immersed. Cells filled with cytoplasm are not simply minuscule bags of water with organelles sloshing around randomly. Rather, cytoplasm is a highly structured viscous fluid that has evolved to allow rapid communication through its unique electromagnetic and quantum properties - through microtubules. The human body is thus possessed of collective quantum effects throughout its entirety, including, most importantly, the central nervous system and the brain. The implication is that vast quantities of information about the state of the organism and its environment can be absorbed and reemitted by single photons.

Non-local Communication. A second inherent property of microtubules is the quantum phenomenon of non-local communication, or so called "action-at-a-distance." Peter Marcer and Walter Schempp (1997) described microtubule signal propagation within the human body as 
occurring instantaneously. Non-local communication is implicated in many subjectively reported altered-state experiences such as neardeath life reviews. Such reports are not in accord with classical concepts of linear time and space. The microtubule network may provide the communication mechanism for instantaneous downloading of an entire lifetime of experiences that are "replayed" in a matter of moments, as if the process occurs at a highly accelerated rate. Note that we are not referring to the familiar neurochemical signal propagation within the central nervous system, which travels at approximately 240 miles per hour. Indeed, we are referring to a related, yet clearly distinct, communications network.

Emergent Properties. Finally, consider that emergent, collective, macroscopic properties may arise from a critical level of coherence of quantum events. The laser, a room-temperature Bose-Einstein condensate, is an example. The photons arising from the absorption and reemission of stimulating energy give rise to the collective coherent effect of an intense beam of light of a single wavelength. In this model, microtubules function as pulse-laser signal generators possessing weak electromagnetic fields that cross over between microtubules (Koruga, Hameroff, Withers, Loutfy, and Sundareshan, 1993). Cross-over between any two microtubules situated parallel to one another would result in interference patterns, similar to bands of dark and light. Encoded within the interference patterns would be enormous quantities of holographic information.

It is well known that anesthetics impair the functioning of microtubules, an effect that leads to loss of consciousness (Hameroff, 1994). Such evidence indicates that microtubules may represent the physical structures responsible for the emergence of consciousness. The net effect of countless bundles of neuronal microtubule "cables" would thus be the basis for a profound emergent collective, macroscopic effect: consciousness. Emergence refers to the occurrence of conscious awareness at a critical threshold-resulting from the cumulative effect of countless microtubules acting in a coordinated manner. Consciousness is thus said to result from collective quantum effects that occur in microtubule networks within the central nervous system (Koruga, 1995). Moreover, experimental research links microtubules to bioinformation processes such as memory and learning (Koruga, Hameroff, Withers, Loutfy, and Sundareshan, 1993). For example, in Alzheimer's disease, the cytoskeleton-the microtubule network-becomes entangled. The clinical symptoms of Alzheimer's disease, that is, cognitive defects in 
learning and memory, have been produced in Gilbert Bensimon and Raymond Chermat's study by the drug colchicine, which causes selective destruction of brain microtubules (Bensimon and Chermat, 1991).

Near-death life reviews within the context of this model represent a specific, emergent, quantum effect that is both collective and macroscopic. Such a model provides a basis for understanding the following examples of non-local communication. Dreams contain a great deal of information and meaning that may require hours to order and integrate. Intuitive ideas are often "downloaded" in a fraction of the time they take to decipher and put into sequential order. A research subject of one of the authors (TEB) spoke of "thunderbolts" of information she "received" that took weeks to fully comprehend. A psychotherapy client of the other author (JEC) dreamt prophetically of her current husband ten years prior to their meeting. Although ordinary states of consciousness usually impede such awarenesses, once accessed through nonordinary states, we order the data as it emerges into four-dimensional space-time. "Perhaps our brains (in particular, coherently oscillating cytoskeletal microtubules) discriminate, detect, and serialize synchronistic and non-local ... quantum states which spontaneously and simultaneously emerge (from what Jung referred to as the 'collective unconscious')" (Koruga, Hameroff, Withers, Loutfy, and Sundareshan, 1993, p. 206).

\section{Holographic Memory}

Ervin Laszlo (1995) theorized that memory may be largely stored in a collective, holographic memory field that lies outside the boundaries of the physical body. Memories would then be accessed by the brain from that ambient field rather than being stored primarily within the brain, as classical neurobiology maintains. This accords with the perceptions of clairvoyants who claim to access information contained in the bioenergetic field surrounding each individual, such as personal imagery, and memories of significant events. The empathetic nature of the neardeath life review, where one's thoughts and actions are reviewed from the perspective of others, may also exemplify information retrieval from an ambient field. This ambient field may also relate to Jung's concept of the collective unconscious, a vast pool of archetypal images.

The principle function of the physical brain may be to serve as a mediator for coherent coupling of numerous subsystems (Ho, 1998a). In the language of computer science, it would be comparable to a complex 
operating system and central processing unit, albeit vastly more sophisticated than any computer currently available. Holographic memory, while stored globally, can be accessed locally, just as an image distributed throughout a holographic plate can be reproduced in its entirety by shining a laser through any part of the plate. The organism can be considered as having its own quantum holographic field (for transmission and reception) with which it communicates to the larger universal or collective field. Such a model would allow for the recovery of stored information from the larger field instantaneously and in unlimited quantities. Coherent systems display neither spatial nor temporal separation. Communication from one part to another occurs instantaneously, regardless of the distance (Ho, 1998b). Such memory recovery correlates with the description of many self-reports of near-death life reviews and may provide a realizable mechanism for them. To understand the nature of such field-to- field communication networks, a basic understanding of holography will be useful.

A hologram is an image of a three-dimensional object, created by first splitting a single, coherent laser beam into two beams. The split-off $o b$ ject beam carries the data content, while the reference beam holds the data location. When the two beams later intersect, a complex interference pattern of dark and light bands results. This pattern encodes the surface characteristics and location of the object being illuminated by the object beam. In order to retrieve the entire stored image, the reference beam is shined through any part of the holographic plate. The holographic technique packs data very tightly, storing extensive amounts of information in a small volume. Our next level of technology hopes to achieve holographic memory storage on a single 12-centimeter disk, estimated to hold one terabyte of data, the equivalent of 200 digital video disks (DVDs) (MacNeill, 2001).

Pribram first proposed that the brain stores information encoded as a hologram. Highly organized microscopic structures within the human body have been proposed as holographic storage media, namely, DNA, facilitated by liquid crystalline proteins and intracellular fluids (Marcer and Schempp, 1996). Holographic memory theory applied to living organisms would allow for the storage of vast amounts of information both inside the physical body and, as Laszlo suggested, outside the body in the ambient zero-point field. The zero-point field, described below, pervades the entire physical universe, including intergalactic space as well as the space within all physical matter. The localized field, in and around the body, would thus be coupled with the much larger global field. The empathetic aspect of the near-death life review 
may be one of the most significant examples of holographic memory retrieval. Through this process, the physical brain would effectively have access to a "collective unconscious" with instantaneous communication (Ho, 1998a; Marcer and Schempp, 1997).

\section{The Quantum Hologram}

Quantum physics involves fundamental interactions between physical matter and light. At this level of material reality, particles behave in peculiar ways that are not intuitively obvious, such as spontaneously tunneling through barriers to emerge instantaneously on the other side. The mathematical formalism of quantum physics is the language used to describe the quantum hologram (Marcer and Schempp, 1999). Edgar Mitchell (1999), who has further applied quantum theory to consciousness, stated that the "discovery of the non-local quantum hologram ... provides the first quantum physical mechanism compatible with the macro-scale three-dimensional world as we experience it."

In simple terms, quantum holography can be described as a novel information-processing paradigm that explains human intra- and intercommunication. Such interactions involve processes on all cosmological scales, from subatomic particles to the interstellar (Marcer and Schempp, 1999). Quantum holography requires us to reconsider our classical view of space-time. One functional example of a quantum holographic information retrieval system is the magnetic resonance imaging (MRI) machine that hospitals routinely use to obtain internal images of the human body. Quantum holography models the workings of neurons in the brain and central nervous system, whose microtubules emit single-photon pulses of light. According to this theory, single-photon pulses of light holographically encode information. Nature may thus have evolved its own quantum holographic system, which utilizes fundamental particles of light that may literally communicate with the whole universe. The other structure relevant to quantum holographic communication and memory networks is DNA.

DNA's capacity for virtually unlimited holographic compression of data has been described by Marcer and Schempp (1996). DNA is considered to be a universal medium, or template, for the recording of holographic information. Previously, it was believed that DNA's principle function was to provide a template for an organism's physical structure. However, DNA's main function is currently theorized to be not structural, but rather one of communication. "DNA therefore defines 
the unique spectral signature or set of frequencies onto which potentially the entire experience or history of an organism can be written (and read)" (Marcer and Schempp, 1996, p. 54). The nearly three billion DNA base pairs contained in every human cell can be thought of as an extensive stack of compact disks, each of which possesses enormous data storage capacity. According to this model, DNA's total base pairs comprise a read-write system of virtually unlimited capacity. Yet an organicallybased, quantum holographic memory system requires one additional concept: the zero-point field.

The existence of the electromagnetic zero-point field has been experimentally demonstrated by Steve Lamoreaux (1997). The zero-point field is also referred to as the quantum vacuum, implying that a plenum, or abundance of matter and energy, exists in interstellar space, which was previously believed to be an empty void. All of space, including the voids between atoms within "solid" matter, contains enormous energy potential. Matter and energy are continuously created and annihilated, emerging spontaneously out of, and disappearing back into, the zeropoint field. It is now widely accepted by physicists that the quantum vacuum is the underlying source of all matter and energy in the universe. Theoretically the energy potential of the vacuum is enormous, perhaps exceeding that of nuclear energy potential (Sokolov, 1996).

Marcer and Schempp (1997) described a quantum model of self-organization from the dynamic quantum vacuum by means of an emitter/absorber model of holography. As each particle emits and absorbs packets of energy or information, propagated through the medium of the zero-point field, the entire history of the particle is stored and accessible. The zero-point field has been proposed as the memory storage medium for all particle interactions, including interactions on a macroscopic scale, such as human memory of life events. Based on the holographic principle, long-term memory retrieval from the ambient zero-point field occurs when interference patterns are reconverted into the image of those objects or events originally recorded (Laszlo, 1995). In this model, the brain and central nervous system are viewed not as memory locations themselves, but rather as organic processes that interact directly with the zero-point field on a quantum level. David Lorimer has intuitively observed that near-death life reviews require "an interconnected web of creation, a holographic mesh in which the parts are related to the Whole and through the Whole to each other by empathetic resonance" (Lorimer, 1990, p. 22). For the first time, a scientifically rigorous biomechanical model is available through which this process can be described. 
Perhaps the most significant finding to come out of this body of research is the application of quantum mechanical theory to the macroscopic world perceivable by the physical senses, as summarized by Mitchell (1999): "Further, recognition that the quantum hologram is a macro-scale, non-local, information structure described by the standard formalism of quantum mechanics extends quantum mechanics to all physical objects including DNA molecules, organic cells, organs, brains, and bodies."

\section{Conclusion}

According to current memory research, it is implausible that one's life, replete with minute details, can be remembered in its entirety, let alone reviewed in a matter of seconds. Such life reviews become even less credible if they include perceptions of those same events as experienced through the senses of others. Yet vividly empathic life reviews are commonly reported during near-death experiences. Moreover, such experiences are often subjectively described as occurring "outside of time and space," which is consistent with the concept of instantaneous communication. Previously there has been no non-dualistic, scientific mechanism for explaining how such communication can occur in the physical human body. Quantum holographic theory may afford an explanation for the physiological mechanism of near-death life reviews.

Quantum holography provides a theoretical model within which the human body may function as a non-local communication network. Microtubules and DNA within the liquid crystalline body are the quantum-scale structures and physical mechanisms required for this level of communication, in terms of (a) the sheer amount of sensory information and information recall, as well as (b) virtually instantaneous information recall. The zero-point field may further provide the medium within which unlimited amounts of information may be written and retrieved by quantum mechanical processes. The vast amount of sensory information and recall necessary for near-death life reviews would thus be realizable.

While the microtubule and DNA communication network would provide the means for an individual's memories, mediation by means of the zero-point field would serve to explain (c) the review of events from the perspective of those with whom the individual had interacted. The zero-point field is not only the source of all energy and matter, but the continuum through which non-local communications may occur across 
any distance. The zero-point field, being comprised of continuous quantum energy fluctuations, which are inherently non-local, would allow for instantaneous communication. This would allow sufficient "time" to elapse during the NDE for the entire life review to complete itself, despite the relatively short duration-in linear time - of NDEs. Moreover, a coherent zero-point field would be equally accessible to every human. Non-local communications mediated by means of the zero-point field would allow near-death experiencers to perceive their actions from the perspective of those with whom they interact, and so forth, until potentially "all lives being lived on this planet" are reviewed.

\section{References}

Arnette, J. (1999). The theory of essence. III. Neuroanatomical and neurophysiological aspects of interactionism. Journal of Near-Death Studies, 14, 73-101.

Aspect, A., Dalibard, J., and Roger, G. (1982). Experimental test of Bell inequalities using time-varying analyzers. Physical Review Letters, 49, 1804.

Audain, L. (1999). Near-death experiences and the theory of the extraneuronal hyperspace. Journal of Near-Death Studies, 18, 103-115.

Becker, C. B. (1995). A philosopher's view of near-death research. Journal of Near-Death Studies, 14, 17-28.

Begley, S. (1996, July 15). You must remember this. Newsweek, p. 64.

Bensimon, G., and Chermat, R. (1991). Microtubule disruption and cognitive defects: Effect of colchicine on learning behavior in rats. Pharmacology, Biochemistry, and Behavior, 38, 141-145.

Colli, J. E. (2001). Angels and aliens: Encounters with both near-death and UFOs. Presented at the International Association of Near-Death Studies 2001 International Conference, Seattle, WA.

Cotton, P. (1994). Medical news and perspectives: Biology enters repressed memory fray. Journal of American Medical Association, 272, 1725-1726.

Fabiani, M., Stadler, M. A., and Wessels, P. M. (2000). True but not false memories produce a sensory signature in human lateralized brain potentials. Journal of Cognitive Neuroscience, 12, 941-949.

Fröhlich, H. (1988). Theoretical physics and biology. In H. Fröhlich (ed.), Biological coherence and response to external stimuli (pp. 1-24). Berlin, Germany: SpringerVerlag.

Gauthier, I., Hayward, W. G., Tarr, M. J., Anderson, A. W., Skudlarski, P., and Gore, J. C. (2002). BOLD activity during mental rotation and viewpoint-dependent object recognition. Neuron, 34, 161-171.

Goswami, A. (1993). The self-aware universe: How consciousness creates the material world. New York, NY: Tarcher/Putnam.

Greene, F. G. (1981). A glimpse behind the life review. Journal of Religion and Psychical Research, 4, 113-130.

Hameroff, S. (2001). Feasibility of macroscopic quantum mechanisms in the brain. Retrieved April 18, 2002, from the University of Arizona course "Consciousness at the Millennium: Quantum Approaches to Understanding the Mind" Web site: http://www.consciousness.arizona.edu/quantum/week7a.htm

Hameroff, S. (1994). Quantum coherence in microtubules: A neural basis for emergent consciousness? Journal of Consciousness Studies, 1, 91-118. 
Hirano, I., and Hirai, N. (1986). Holography in the single-photon region. Applied Optics, $25,1741-1742$.

Ho, M-W. (1999, October 2). Coherent Energy, Liquid Crystallinity and Acupuncture [Talk presented to the British Acupuncture Society]. Retrieved April 18, 2002, from the Institute of Science in Society Web site: http://ratical.org/coglobalize/MaeWanHo/acupunc.html

Ho, M-W. (1998a). Organism and psyche in a participatory universe. In D. Loye (ed.), The evolutionary outrider. The impact of the human agent on evolution: Essays in honour of Ervin Laszlo (pp. 49-65). Westport, CT: Praeger.

Ho, M-W. (1998b). The rainbow and the worm: The physics of organisms. Singapore: World Scientific Publishing.

Koruga, D. (1995). Information physics: In search of a scientific basis of consciousness. In D. Koruga and D. Rakovic (eds.), Consciousness: Scientific challenge of the 21st century (pp. 243-261). Belgrade, Serbia: European Centre for Peace and Development (ECPD) of the United Nations University for Peace.

Koruga, D., Hameroff, S., Withers, J., Loutfy, R., and Sundareshan, M. (1993). Fullerene $C_{60}$ : History, physics, nanobiology, nanotechnology. New York, NY: North-Holland.

Krishnan, V. (1996). Misidentified flying objects. [Letter]. Journal of Near-Death Studies, $14,287-290$.

Lamoreaux, S. (1997, January 6). Demonstration of the Casimir force in the 0.6 to 6 micron range. Physical Review Letters, 78(1), 5-8.

Laszlo, E. (1995). The interconnected universe: Conceptual foundations of transdisciplinary unified theory. Singapore: World Scientific Publishing.

Loftus, E. F. (1997, September). Creating false memories. Scientific American, 277(3), 70-75.

Loftus, E. F., and Hoffman, H. G. (1989). Misinformation and memory: The creation of new memories. Journal of Experimental Psychology: General, 118, 100-104.

Loftus, E. F., and Loftus, G. R. (1980). On the permanence of stored information in the human brain. American Psychologist, 35, 409-420.

Lorimer, D. (1990). Whole in one: The near-death experience and the ethic of interconnectedness. London, England: Arkana.

MacNeill, J. (2001, May). Holographic memory: Laser beams store data in three dimensions. Technology Review, pp. 96-97.

Marcer, P. J., and Schempp, W. (1996). A mathematically specified template for DNA and the genetic code in terms of the physically realizable processes of quantum holography. In A. M. Fedorec and P. J. Marcer (eds.), Proceedings of the Greenwich Symposium on Living Computers (pp. 45-62). Wiltshire, England: British Computer Society.

Marcer, P. J., and Schempp, W. (1997). Model of the neuron working by quantum holography, Informatica, 21, 519-534.

Marcer, P. J., and Schempp, W. (1999). Quantum holography: The paradigm of quantum entanglement. In D. M. Dubois (ed.), Computing anticipatory systems: CASYS '98, Second International Conference (pp. 461-467). College Park, MD: American Institute of Physics.

Marshall, I. (1989). Consciousness and Bose-Einstein condensates. New Ideas in Psy. chology, 7(1), 73-83.

Mitchell, E. (1999). Nature's mind: The quantum hologram. Retrieved April 18, 2002 from the National Institute for Discovery Science Web site: http://www.nidsci.org/articles/naturesmind-qh.html

Moody, R. (1975). Life after life. Covington, GA: Mockingbird Books.

Morse, M., and Perry, P. (1992). Transformed by the light: The powerful effect of near-death experiences on people's lives. New York, NY: Villard.

Nimtz, G. (1998). Superluminal signal velocity. Annals of Physics (Leipzig), 7, 618-624.

Nimtz, G. (1999). Evanescent modes are not necessarily Einstein causal. European Physical Journal B, 7, 523-525. 
Noyes, R., and Kletti, R. (1977). Panoramic memory: A response to the threat of death, Omega, 8, 181-194.

Penfield, W. (1969). Consciousness, memory, and man's conditioned reflexes. In K. H. Pribram (ed.), On the biology of learning (pp. 127-168). New York, NY: Harcourt, Brace and World.

Penfield, W. (1975). The mystery of the mind: A critical study of consciousness and the human brain. Princeton, NJ: Princeton University Press.

Popp, F., Li, K., Nagl, W., and Klima, H. (1983). Indications of optical coherence in biological systems and its possible significance. In H. Fröhlich and Kremer (Eds.), Coherent excitations in biological systems (pp. 117-122). New York: Springer-Verlag.

Pribram, K. (1969, January). The neurophysiology of remembering. Scientific American, $220(1), 73-86$.

Puthoff, H. E. (1996). CIA initiated remote viewing program at Stanford Research Institute. Journal of Scientific Exploration 10, 63-76.

Radin, D. (1997). The conscious universe: The scientific truth of psychic phenomena. San Francisco, CA: HarperSanFrancisco.

Ring, K., and Valarino, E. E. (1998). Lessons from the light: What we can learn from the near-death experience. New York, NY: Plenum/Insight.

Rosenthal, M. H., and Larson, C. P. (1978). Protection of the brain from progressive ischemia. Western Journal of Medicine, 128, 145.

Sabom, M. (1998). Life and death: One doctor's fascinating account of near-death experiences. Grand Rapids, MI: Zondervan.

Schacter, D. L., Reiman, E., Curran, T., Yun, L. S., Bandy, D., McDermott, K. B., and Roediger, H. L. (1996). Neuroanatomical correlates of veridical and illusory recognition memory: Evidence from positron emission tomography. Neuron, 17, 267-274.

Sokolov, I. (1996). The Casimir effect as a possible source of cosmic energy. Physics Letters A, 223, 163-166.

South wick, S. M., Morgan, C. A., Nicolaou, A. L., and Charney, D. S. (1997). Consistency of memory for combat-related traumatic events in veterans of Operation Desert Storm. American Journal of Psychiatry, 154, 173-177.

Stapp, H. P. (1994). Theoretical model of a purported empirical violation of the predictions of quantum theory. Physical Review A, 50(1), 18-22.

Stuchebrukhov, A. A. (1996). Tunneling currents in electron transfer reaction in proteins. II. Calculation of electronic superexchange matrix element and tunneling currents using nonorthogonal basis sets. Journal of Chemical Physics, 105, 10819-10829.

van der Kolk, B. A., and Fisler, R. (1995). Dissociation and the fragmentary nature of traumatic memories: Review and experimental confirmation. Journal of Traumatic Stress, 8, 505-525.

van der Kolk, B. A., McFarlane, A. C., and Weisaeth, L. (Eds.). (1996). Traumatic stress: The effects of overwhelming experience on mind, body and society. New York, NY: Guilford Press.

van der Kolk, B. A., and van der Hart, O. (1991). The intrusive past: The flexibility of memory and the engraving of trauma. American Imago, 48, 425-454.

Wade, J. (1996). Changes of mind: A holonomic theory of the evolution of consciousness. Albany, NY: State University of New York Press.

Wagenknecht, H. A., Rajski, S. R., Pascaly, M., Stemp, E. D., and Barton, J. K. (2001). Direct observation of radical intermediates in protein-dependent DNA charge transport. Journal of the American Chemical Society, 123, 4400-4407. 